\title{
El papel de las AMPA en los centros escolares: actuaciones y retos ${ }^{1}$ \\ The role of parent's associations (AMPA): actions and challenges
}

\section{Olga Bernad Cavero y Núria Llevot Calvet ${ }^{2}$}

\section{Resumen}

Partiendo de una etnografía realizada durante nueve meses en 11 centros escolares de Cataluña de educación infantil y primaria y secundaria obligatoria (ESO) sobre la participación de las familias, este artículo se centra en la participación colectiva de las familias a través de las asociaciones de madres y padres de alumnos (AMPA) y analiza el papel de esta entidad en los centros, así como señala los puntos débiles y las fortalezas desde el punto de vista de los propios actores. Se concluye que la buena gestión y funcionamiento de las AMPA es uno de los factores clave en la conformación de dinámicas positivas de relación y participación de las familias, a la vez que tendrían que redefinir su papel y funciones en el contexto actual.

\section{Palabras clave}

Escuela, familias, puntos débiles, fortalezas, actuaciones, retos, asociaciones de madres y padres.

\section{Abstract}

Based on an ethnography conducted during 9 months in 11 schools of Catalonia, pre-school and primary education and compulsory secondary education (ESO), concerning the participation of families, this article focuses on the collective participation of families through parents' associations (AMPA) and analyzes the role of this entity in the centers, pointing out the weaknesses and strengths from the viewpoint of the actors. It concludes that good management and functioning of the AMPA is one of the key factors in shaping positive dynamics of relationship and family involvement, but AMPA would have to redefine its role and functions in the current context.

\section{Keywords}

School, families, weaknesses, strengths, activities, challenges, parents'association.

\footnotetext{
1 Este artículo se inscribe dentro del proyecto de investigación "Familias y escuelas. Discursos y prácticas cotidianas sobre la participación en la educación obligatoria" (Ministerio de Economía y Competitividad; referencia EDU2012-32657), en el cual participan las universidades de Lleida, Zaragoza, La Rioja e Islas Baleares. Para facilitar la lectura, utilizaremos el género masculino para referirnos a ambos géneros, el masculino y femenino.

2 olga@geosoc.udl.cat; nllevot@pip.udl.cat
} 


\section{Introducción}

Actualmente se considera la implicación de las familias en la acción educativa de los centros escolares como uno de los factores que influyen en el éxito escolar de los niños y niñas y uno de los indicadores de calidad del sistema educativo.

La participación de los padres y madres en los centros escolares repercute en los alumnos, en las familias y en el profesorado. Por ejemplo, Epstein (2001) destaca la mejoría de los resultados académicos, actitudes y comportamiento de los alumnos; Bolívar (2006) y Grant y Ray (2013) indican los beneficios en las actuaciones docentes y en el funcionamiento del centro, y Kherroubi (2008) el incremento de capital social y mejores competencias parentales y actitudes de las familias hacia la escuela. Pero también redunda en la calidad ${ }^{3}$ del sistema educativo y del centro.

El artículo 27 de la Constitución Española de 1978 reconoce el derecho de los padres y madres a participar en la educación de sus hijos e hijas, y las leyes educativas posteriores lo han desarrollado (Garreta, 2008; Giró, Mata, Vallespir y Vigo, 2014; Frías del Val, 2014). Debido a las vicisitudes histórico-políticas que ha vivido España en el siglo xx, no es hasta principios de la década de los 70 cuando se genera la conciencia social de la importancia de la participación activa de los padres y madres en la educación de los hijos, en un momento histórico en el que, no por casualidad, se crean las primeras asociaciones de padres y federaciones, y en el que progenitores y docentes, conjuntamente, luchan por una escuela pública de calidad.

Se reconoce a las familias unos derechos individuales y colectivos (para ampliar, ver Vega, Navaridas, Giró, Muñoz y Blandini, 2012). Así tienen derecho a: participar como padres y madres de sus propios hijos e hijas (que en el momento que empiezan su escolaridad adoptan el nuevo rol de alumnos y alumnas), escoger el centro escolar, estar informadas por el centro y velar por la defensa de sus intereses, etc. Pero como miembros de una sociedad democrática, también tienen derecho a participar a través de sus representantes en los órganos de gestión del centro, especialmente en el Consejo Escolar (y mediante las asociaciones de madres y padres), defendiendo los intereses del conjunto del alumnado (y sus familias) del centro. Por otra parte, como señala Kherroubi (2008) por ejemplo, la participación de los padres en la escuela es efímera, ya que tiene lugar solamente mientras los hijos están matriculados en el centro.

En los últimos años, las políticas educativas de la mayoría de los países europeos (España entre ellos) ponen el acento en la implicación de las familias en la acción educativa, resaltando que la participación colectiva a través de los órganos formales reconocidos tradicionalmente es un elemento más, pero insuficiente por sí solo, para conseguir el éxito educativo del alumnado. Se percibe la participación de los padres como un indicador de calidad de la educación y de los centros.

Se suelen distinguir dos modalidades de participación: individual y colectiva. Y dos ámbitos: formal e informal. Las familias participan individualmente, por ejemplo, cuando solicitan una entrevista con el tutor, y colectivamente, si forman parte de la asociación de madres y padres de alumnos del centro $(\mathrm{AMPA})^{4}$. En el ámbito formal, se puede participar asistiendo a la reunión de inicio de curso o a la asamblea del AMPA, por ejemplo. Y en el informal, hablando con el profesorado en las entradas y salidas del centro. Sin embargo, la literatura y la realidad cotidiana de nuestros centros educativos constatan la baja participación de las familias, sobre todo en los órganos reconocidos legalmente: el AMPA y el Consejo

3 En su informe sobre la calidad educativa, la Comisión Europea (2000) considera la participación de las familias como el indicador 12 de calidad en la educación.

4 Otra denominación para referirse a esta asociación, sobre todo en el caso de los centros privados con concierto, es AFA (Asociación de Familias de Alumnos). 
Escolar (Garreta, 2008, 2016; Vallespir, Rincón y Morey, 2016), donde, al mismo tiempo, es poco valorada. Para ilustrar la poca valoración que recibe la participación en estos órganos, nos remitimos a un estudio dirigido por Jordi Garreta (2012) en el que se realizaron encuestas durante el año 2010 a equipos directivos de centros educativos públicos de Cataluña. Entre los elementos que muestran una implicación adecuada ${ }^{5}$ por parte de las familias, se cita la inscripción en el AMPA $(15,1 \%)$ y, en cambio, la participación en la junta del AMPA y en el Consejo Escolar es mencionada por menos del 1\% de la muestra. En la misma línea, el estudio de Martínez González, Rodríguez y Gimeno (2010) indica una elevada participación en cuanto a la inscripción (76\%), pero poca colaboración en su organización (78\%) y en la asistencia a las actividades organizadas (49\%).

Este artículo se centra en la participación colectiva de las familias mediante el AMPA y analiza el papel asignado (y no asignado), en los centros de educación infantil y primaria y secundaria obligatoria de Cataluña sostenidos con fondos públicos, a la participación de las familias y al funcionamiento de los centros. Se hace especial hincapié en las actuaciones que realizan y a quiénes van dirigidas, y en los retos que se plantean, señalando sus puntos débiles y sus fortalezas desde el punto de vista de los actores. Para ello se parte de los resultados de una etnografía escolar realizada en 11 centros escolares de Cataluña, públicos y privados con concierto, sobre la participación de las familias en la educación obligatoria. Pero antes de mostrar el trabajo empírico, se hará un breve recorrido sobre el movimiento de padres, desde la constitución de las primeras asociaciones hasta el momento actual, punto de inicio de la etnografía.

\section{Origen y evolución del movimiento de padres y madres}

En España, debido a las particulares circunstancias históricas, los orígenes de las AMPA se remontan a finales de la década de los años 60 y principios de los 70, cuando cristalizan diferentes movimientos populares (movimiento vecinal, de padres, de renovación pedagógica, etc.) que reivindican la mejora de las condiciones de vida de los ciudadanos, y concretamente en el campo educativo, una escuela para todo el mundo, gratuita, participativa y de calidad, estabilidad del profesorado, etc. A pesar de que el Decreto de 1964 permitía la creación de las APA, hasta entonces la participación y colaboración de las familias en los centros dependía de las relaciones, las necesidades y demandas que hacían los centros.

En esa época las necesidades en el campo educativo son muy importantes. Son años de considerable aumento de la natalidad (baby boom), de grandes desplazamientos de población del ámbito rural al urbano, de concentración en las grandes ciudades españolas y sus áreas metropolitanas. A estas necesidades cuantitativas de plazas escolares se une un movimiento por la calidad de la enseñanza y por la recuperación de movimientos de innovación pedagógica y social que apenas pudieron empezar a expresarse en la enseñanza durante la II República y fueron reprimidos por el franquismo (Garreta, 2008: 58).

Estos factores favorecen la fundación, en los años 70, de las AMPA en muchos centros en todo el Estado, con lo que el Movimiento de Padres crece en importancia y se acaba estructurando, entre los últimos años de esta década e inicios de los 80 , con la formación de las federaciones y confederaciones a escala autonómica y estatal.

Con la llegada de la democracia, se reconoce legislativamente el derecho de las familias a asociarse y a participar en los centros educativos donde cursan estudios sus hijos e hijas. En 1972 se creó en Barcelona la primera coordinadora de APA. Más tarde, este movimiento se fue extendiendo a otras poblaciones y comarcas, con unos objetivos que sintonizaban con el contexto político del momento: estabilidad del

5 Los elementos considerados más importantes son: la asistencia a las tutorías (68.6\%) y a las reuniones convocadas por el centro (31\%); el interés por lo que hacen sus hijos $(30.6 \%)$ y el seguimiento de sus deberes $(27.3 \%)$. 
profesorado, escuela gratuita, escuela catalana, escuela con participación de padres y madres, etc. Todo ello cristalizó en 1975 con la creación de la FAPAC (Federación de Asociaciones de Madres y Padres de Alumnos de Cataluña).

En los años 80 y 90 se producen algunos cambios sociales que repercuten en las escuelas, como el descenso de la natalidad, pero a la vez la Administración empieza a primar criterios de racionalización económica que comportan que las AMPA empiecen a asumir servicios que hasta ese momento habían recaído en la Administración, como el de comedor o las actividades extraescolares. A partir de este momento, el movimiento de padres empieza a perder fuerza, en parte debido a que algunos de sus líderes pasan a ocupar cargos en la Administración o en instituciones. Actualmente, podemos decir que las AMPA están ampliamente implantadas en los centros escolares y la mayoría de los equipos directivos están interesados en que la asociación esté presente en su centro. Pero con el paso del tiempo, aunque las finalidades iniciales continúen figurando en la declaración de principios de muchas asociaciones, esta capacidad reivindicativa ha pasado a segundo término y la mayoría de AMPA se han convertido en empresas gestoras de servicios como las actividades extraescolares, teniendo algunas una numerosa plantilla de personal a su cargo, y pasando a formar parte de la estructura organizativa de los centros.

A partir de la década de los 90, con la llegada masiva de la nueva inmigración, surgen nuevos retos que tiene que encarar nuestro sistema educativo (incremento repentino del número de alumnos, diversidad de culturas, dificultades lingüísticas, convivencia en los centros, etc.) y que repercuten también en las AMPA. Actualmente, de acuerdo con el modelo del family involvement, se centra la mirada en el papel de estas entidades como colaboradoras en la gobernanza de los centros escolares (pero, eso sí, con un papel secundario) para conseguir una mayor calidad de la educación y, por ende, del centro. Para muchas familias, la oferta de diferentes actividades extraescolares a escoger, comedor con cocina propia en vez de catering, servicio de guardería, etc., es uno de los indicadores de calidad del centro escolar. Pero, a la vez, esto provoca también diferencias entre las distintas AMPA. Hay asociaciones con capacidad para gestionar todos estos servicios y otras, con menos recursos económicos, no lo son tanto. A pesar de que hay un sistema de subvenciones y de becas para las familias con menos recursos, la existencia de estas actividades de pago a cargo de los padres abre una importante barrera en la gratuidad de los centros públicos y, a la vez, en la consideración de su calidad.

En resumen, con la llegada de la Transición se abren vías institucionalizadas de participación y representación política como resultado de la busca de canales de participación que se había iniciado en los últimos años del franquismo y, así, el movimiento de padres gana representatividad institucional y un mayor reconocimiento social, pero este hecho comporta a la vez el freno de la vía reivindicativa y la pérdida de participación directa. Actualmente, aunque el papel de las AMPA goza de reconocimiento institucional y social, como iremos viendo, las asociaciones se enfrentan a importantes retos: supervivencia de la asociación en algunos centros, gobernanza de la asociación, comunicación con las familias, comunicación con los equipos directivos, integración de las familias de origen minoritario, dinamización global de la participación de las familias, etc.

\section{Metodología}

Se parte principalmente de los resultados de un trabajo etnográfico escolar (Serra, 2004) más amplio, realizado durante un mínimo de ocho meses durante los cursos escolares 2013-14 y 2014-2015, en 11 centros de educación infantil y primaria y educación secundaria obligatoria (ESO), de titularidad pública y privada, de la comunidad autónoma de Cataluña. Concretamente, para la etapa de educación infantil y 
primaria se escogieron cinco escuelas públicas, y para la de ESO, tres institutos. Además, se incluyeron tres centros privados con concierto que impartían las etapas de enseñanza obligatoria hasta ESO.

La muestra se escogió de forma no aleatoria, entre centros que destacaban a priori por las buenas relaciones familia-escuela y por sus prácticas participativas. Los centros elegidos se encuentran distribuidos por todo el territorio catalán y responden a diferentes perfiles en cuanto a número de alumnos, ubicación territorial, origen del alumnado, titularidad del centro, etc. Se realizaron entrevistas en profundidad (a miembros del equipo directivo, docentes, representantes de las AMPA y progenitores) y también observaciones en diferentes momentos de la vida diaria del centro, participando incluso en reuniones y actividades diversas.

A continuación, mostramos el cuadro de las 11 etnografías que hemos realizado para este estudio, en el que se describen las principales características de los centros guardando siempre el anonimato que les prometimos.

\section{Tabla I. Etnografías}

\begin{tabular}{|c|c|c|c|c|c|}
\hline Número & $\begin{array}{c}\text { Titularidad } \\
\text { (pública/ privada) }\end{array}$ & $\begin{array}{l}\text { Etapas educativas } \\
\text { analizadas }\end{array}$ & Observaciones & Número alumnos & AMPA \\
\hline $1^{0}$ & Pública & $\begin{array}{l}\text { Infantil } \\
\text { Primaria }\end{array}$ & $\begin{array}{l}\text { Comunidad aprendizaje } \\
\text { Escuela multicultural } \\
\text { urbana }\end{array}$ & 170 & $\begin{array}{l}\text { Escasa implicación de las familias de origen } \\
\text { extranjero }\end{array}$ \\
\hline $2^{0}$ & Privada (con concierto) & $\begin{array}{l}\text { Infantil } \\
\text { Primaria } \\
\text { ESO }\end{array}$ & Urbana & 800 & Colabora en los proyectos del centro \\
\hline $3^{0}$ & Pública & $\begin{array}{l}\text { Infantil } \\
\text { Primaria }\end{array}$ & Escuela rural & 50 & $\begin{array}{l}\text { Colabora en proyectos curriculares } \\
\text { y actividades del centro } \\
\text { Actividades para todo el pueblo } \\
\text { Conflictos entre sectores de familias } \\
\text { Comedor propio }\end{array}$ \\
\hline $4^{0}$ & Privada (con concierto) & $\begin{array}{l}\text { Infantil } \\
\text { Primaria }\end{array}$ & $\begin{array}{l}\text { Escuela multicultural } \\
\text { Urbana }\end{array}$ & 350 & $\begin{array}{c}\text { Se denomina AFA } \\
\text { Poca participación de las familias de origen } \\
\text { extranjero en la junta } \\
\text { Comisiones } \\
\text { Padres delegados de aula } \\
\text { Proyectos en colaboración con otras entidades para } \\
\text { favorecer la convivencia y la participación } \\
\text { de las familias } \\
\text { Colabora en actividades del centro }\end{array}$ \\
\hline $5^{0}$ & Pública & $\begin{array}{l}\text { Infantil } \\
\text { Primaria }\end{array}$ & Urbana & 500 & $\begin{array}{c}\text { Comisiones } \\
\text { Secretaria administrativa } \\
\text { Banco de familias } \\
\text { Colabora en actividades curriculares y proyectos } \\
\text { del centro } \\
\text { Comedor propio }\end{array}$ \\
\hline $6^{0}$ & Pública & $\begin{array}{l}\text { Infantil } \\
\text { Primaria }\end{array}$ & $\begin{array}{l}\text { Escuela nueva creación } \\
\text { Urbana }\end{array}$ & 330 & $\begin{array}{c}\text { Comisiones } \\
\text { Colabora en proyectos y actividades del centro }\end{array}$ \\
\hline $7^{0}$ & Pública & $\begin{array}{l}\text { Infantil } \\
\text { Primaria }\end{array}$ & $\begin{array}{l}\text { Escuela nueva creación } \\
\text { Consejo de familias } \\
\text { Consejo de niños y niñas } \\
\text { Urbana }\end{array}$ & 600 & $\begin{array}{c}\text { Comisiones } \\
\text { Padres delegados de aula } \\
\text { Proyectos propios abiertos al municipio } \\
\text { Colabora en proyectos curriculares } \\
\text { y actividades del centro } \\
\text { Banco de familias } \\
\text { Comedor propio }\end{array}$ \\
\hline $8^{0}$ & Pública & ESO & Urbana & 600 & Colabora en proyectos y actividades del centro \\
\hline $9^{0}$ & Privada (con concierto) & ESO & $\begin{array}{l}\text { Cooperativa de padres } \\
\text { Urbana }\end{array}$ & 869 & $\begin{array}{l}\text { AMPA sustituida en funciones por una comisión } \\
\text { delegada de actividades del Consejo Rector } \\
\text { Comedor propio }\end{array}$ \\
\hline
\end{tabular}




\begin{tabular}{ccccc}
\hline $10^{\circ}$ & Pública & USO & 800 & $\begin{array}{c}\text { Técrico administrativo } \\
\text { Gestiona algunos proyectos del centro y colabora en } \\
\text { actividades }\end{array}$ \\
\hline $11^{\circ}$ & Pública & Urbana & 850 & $\begin{array}{c}\text { Colabora en proyectos y actividades del centro } \\
\text { Las familias no pagan cuota }\end{array}$ \\
\hline
\end{tabular}

Fuente: elaboración propia

\section{Las AMPA: actuaciones y retos}

$\mathrm{Al}$ igual que en los centros, en las AMPA se observan dinámicas de funcionamiento y organización y relaciones de poder que perfilan sinergias propias que hacen que cada asociación sea única. Así, hay asociaciones con miembros activos que funcionan por comisiones, que tienen una estructura de funcionamiento propia que favorece la participación de las familias y la apertura al entorno, y en cambio en otras la junta directiva está formada solamente por los cargos que dicta la normativa (presidente, secretario y tesorero) y su permanencia discurre a expensas del apoyo de la Dirección del centro.

Las AMPA realizan una gran variedad de funciones, que cambian dependiendo de los centros:

- Gestionar servicios y organizar actividades para el alumnado (en colaboración con el centro): servicio de comedor escolar, servicio de guardería (en los centros de infantil y primaria), actividades extraescolares deportivas y culturales para atender las demandas de las familias y los alumnos, socialización (reutilización) de libros y material, colonias, talleres, excursiones, etc.

Las extraescolares, la casa de verano, unas colonias que este año se están intentando sacar adelante, que costará mucho porque la gente no tiene dinero. (CPCA114A) ${ }^{6}$.

- Organizar actividades educativas y lúdicas para las familias, para favorecer la convivencia y la implicación de las familias en la educación de sus hijos e hijas: conferencias, talleres, cursos de formación (informática, catalán, etc.), salidas de fin de semana y excursiones, cursos de baile, propuesta de actividades lúdico-educativas conjuntas, etc.

El AMPA, el AMPA del Centro, es un AMPA muy activo y montan conferencias útiles para los padres, para que entiendan un poco más a sus hijos. Adolescentes, las drogas... (IPUPS214B).

- Organizar actividades para toda la comunidad educativa para favorecer un buen clima de convivencia y la cohesión social (en colaboración con el centro y con entidades del entorno): fiestas y celebraciones, jornadas interculturales, salidas y excursiones, conferencias, tertulias, etc.

El objetivo es mejorar las relaciones; la excusa, el aprendizaje para la lengua catalana... las familias que quieran se podrán reunir alli para charlar, aprender catalán, compartir experiencias... (CРCPP314B).

- Prestar ayuda a familias en situación económica desfavorecida: ayudas económicas para pagar cuotas del servicio de comedor, fraccionamiento de pagos (por ejemplo, de salidas o actividades extraescolares), becas a alumnos, etc.

Incluso ahora, en tiempo de crisis, cuando ha babido algún alumno que no ba podido costearse sus actividades, por razones más que obvias, es el AMPA el que se encarga de canalizar cómo lo hace. Y no me estoy refiriendo a libros, sino que un alumno que sus padres están en el paro pues que pueda ir a todas las excursiones y ellos ya se encargan

6 Los acrónimos identificativos de las entrevistas se han construido de la siguiente forma: primera parte indica si es centro de infantil y primaria (C) o instituto (I); segunda parte si es de titularidad pública (PU) o privada con concierto (PC); la tercera parte indica quién es el entrevistado (D director; S secretario; PP profesor infantil; PP1 docente de primer o segundo curso de primaria; PP2 docente de tercer o cuarto curso y PP3 de tercer curso; PS1, PS2 docente de primero o segundo de secundaria o tercero y cuarto; A1 representante AMPA de primaria y A2 de secundaria obligatoria; FPO familiar de alumnado de infantil, FP1 de primero y segundo curso de primaria, FP2 de tercero y cuarto, FP3 de quinto y sexto; FS1 familiar de primero y segundo de secundaria obligatoria y FS2 de tercero y cuarto; TA técnico de AMPA); la cuarta parte del acrónimo indica el curso escolar en que se hizo la entrevista (2013-14 sería 14A, 2014-15 14B). 
de: 'Bueno, pues mira, nos ayudarás a forrar los libros o lo que sea'. Y ellos ponen el dinero. Es un AMPA muy implicada que no desatiende las preocupaciones de... (IPUPS214B).

- Ayudar a satisfacer las necesidades del centro a través de: compra de material, contratación de profesionales (monitores para la biblioteca, psicopedagogos, técnicos auxiliares...), ayudas económicas (para sufragar gastos no cubiertos por las Administraciones como obras y mejoras de las infraestructuras, proyectos, excursiones del alumnado, conferenciantes...), bancos de familias voluntarias (para colaborar en tareas y actividades pedagógicas y en la mejora de las instalaciones), colaboración en actividades del centro y en la acogida de las nuevas familias (día de puertas abiertas, fiestas...), etc.

El AMPA intenta ayudar a llegar donde la escuela no llega [...]: monitoras de comedor de patio, comprar ordenadores para la escuela, palas para la actividad de pádel. (CPUFP114A).

- Defender los intereses del centro y/o de las familias ante las administraciones (pedir y reclamar servicios, obras y arreglo de las instalaciones e infraestructuras, recursos humanos, elevar quejas sobre el profesorado, etc.).

El año pasado medio amenazaron de que, como no teniamos suficientes alumnos, igual nos sacaban el comedor [...] Sí, se creó una comisión, para hablar con el Consejo comarcal, con el ayuntamiento, intentar buscar alternativas, y tal [...]. Pero bueno, un poco hacer de puente entre... porque claro, si el Consejo comarcal va cualquier padre también se te quejan. En cambio, si va gente como AMPA, te atienden diferente. (CPUA114B).

- Servir de puente entre el centro y las familias (defender los intereses de los padres ante la dirección del centro, mediar en las demandas, realizar sugerencias pedagógicas o de gestión, servir de instrumento a la Dirección para conocer qué piensan las familias del centro, etc.)

Porque habia donaciones [...], el día de Sant Jordi se regalaba una rosa a todas las maestras. Y este año dijimos, ¿por qué tenemos que hacer esto?, ¿no? Todas tienen marido, que le regalará... (CPCA114AI).

- Participar en los órganos de gestión del centro: Consejo Escolar, Consejo de Familias, Consejo de Padres y Madres Delegados de Aula, etc. Subrayamos que a pesar de que prescriptivamente un miembro del AMPA tiene que estar en el Consejo Escolar, esta función apenas es mencionada por los entrevistados, en cambio se cita con mayor frecuencia la asistencia a las reuniones de madres y padres delegados de aula.

El representante del AMPA coordina la reunión y luego están los delegados de clase. Pero cuesta mucho que las familias se centren en los temas generales. (CIPCD14B).

- Favorecer y promover la participación de las familias a través de: comisiones, bancos de familias, proyectos, actividades, etc.

Sí, a veces hay tareas de mantenimiento de la escuela, pues que también se pide que algún padre que se pueda dedicar al tema o que trabaje en el ámbito de la necesidad que tiene la escuela, pues se pide a ver si puede participar. Y de hecho ha habido varias cosas que se han hecho así. (CPUA114A).

- Favorecer y promover la comunicación con las familias a través de los canales de comunicación del AMPA.

Y entonces, ¿qué hacemos? Pues la AFA tiene un apartado, aqui delante [...] Quiero decir que esta parte, es la parte de información... (CPCD14A).

Éstas son las principales funciones que desarrollan las AMPAS en los centros estudiados. A continuación, analizaremos los retos a que se enfrentan actualmente, señalando asimismo sus fortalezas. 


\section{Retos de las AMPA}

Un aspecto a analizar es cómo ha repercutido en las AMPA la crisis socioeconómica que ha atravesado nuestro país en los últimos años. Uno de los puntos fuertes señalados por los entrevistados y constatado por otros estudios (Garreta, 2008, 2016), es la prestación de servicios y organización de actividades, dirigidas a satisfacer las necesidades y demandas de los alumnos, de las familias y de los centros. Estas entidades se financian principalmente con las cuotas de los socios, subvenciones de las Administraciones (de los ayuntamientos, principalmente), actividades extraescolares y la distribución de libros de texto. Y aunque, en general, las actividades que organizan las AMPA de nuestro estudio se dirigen a todas las familias del centro (y no solo a las familias socias) e incluso en algún caso al entorno, hay que tener en cuenta que, en los últimos años, en muchas asociaciones ha disminuido el número de familias socias y el de alumnos que realizan actividades extraescolares, a la vez que han aumentado las cuotas impagadas en los servicios de comedor, por ejemplo. También las administraciones "han recortado" las subvenciones y algunas asociaciones tienen problemas de liquidez económica. Si bien una queja generalizada, tanto por parte del profesorado (Andrés y Giró, 2016) como de los representantes asociativos, es la minoración de recursos económicos de que disponen las asociaciones, en algunos centros ha servido sin embargo de revulsivo para buscar soluciones imaginativas en colaboración con el centro para dar respuesta a nuevas necesidades (Giró y Andrés, 2014, 2015) que han ido surgiendo. Por ejemplo, para paliar los recortes en recursos humanos y materiales, en algunos centros se ha aprovechado su capital humano y se han implementado los bancos de familias (generalmente se trata de una ayuda canalizada y "controlada" por la dirección y el profesorado), ya sea como personal de apoyo en las actividades lectivas de las aulas y logísticas (como acompañar a los niños a la piscina) o como mano de obra para mejorar las instalaciones. Pero este tipo de actuaciones también ha servido para renovar paulatinamente el funcionamiento de las asociaciones y de los centros y acercar algunas familias a la asociación y a la escuela, invitándolas a participar.

Por otra parte, también las AMPA han tenido que hacer frente a necesidades por parte de las familias. Algunas, debido a su situación socioeconómica, se han visto imposibilitadas para hacer frente al pago de los servicios de comedor o extraescolares o incluso la cuota de socio. Y las AMPA se han visto obligadas a buscar soluciones, como fraccionar los pagos, becar algunos alumnos o adelantar las cuantías del servicio de comedor, hasta que llegan las becas o subvenciones. En algunos centros, la asociación ha emprendido algunas iniciativas dirigidas a las familias del municipio en colaboración con entidades del tercer sector, como recogida de juguetes y alimentos, extraescolares y charlas y actividades culturales y deportivas gratuitas a cargo de voluntarios (padres o personas externas), etc. Para hacer frente a la disminución de familias socias y por tanto de los ingresos procedentes de las cuotas (que comporta a su vez menores recursos económicos y materiales para el centro, aspecto que se cita persistentemente en las etnografías), en algunos centros la Dirección ha optado por incluir la cuota de socio en la matrícula de los alumnos. Por otra parte, en algún centro público, a raíz de la implicación de las AMPA en las movilizaciones en protesta contra los recortes en educación y en defensa de una educación de calidad, se ha tomado conciencia por parte de algunos representantes asociativos del derecho social de las familias a participar en los centros, reclamando una enseñanza de calidad y un funcionamiento más democrático y su participación activa, más allá de ser meros prestadores de servicios, en la construcción del proyecto de escuela, siendo conscientes sin embargo de los límites impuestos.

Si que ha cambiado un poco la colaboración con la escuela, que antes los padres no colaborábamos con tareas educativas dentro de la escuela y bace un par de años, a raíz de la crisis si que nos han pedido a ver si babia padres que quisieran colaborar, dar una mano con tareas que la escuela no llega. (CPUA114A). 
Ahora estamos en época de crisis, pero en épocas buenas yo sé que el AMPA incluso... Era un AMPA muy potente, incluso a nivel económico. Con pocas aportaciones, pero incluso financiaban proyectos. Incluso abora en tiempo de crisis cuando ba habido algún alumno que no ha podido costearse sus actividades, por razones más que obvias, es el AMPA la que se encarga de canalizar cómo lo hace. (IPUPS214B).

Otro reto prioritario es la comunicación con las familias (consultar: Garreta, 2015; Garreta y Llevot, 2015; Macià, 2016). En algunos centros es uno de sus puntos débiles, pero en otros es una de sus fortalezas, aunque en todos los casos se piensa que hay que seguir trabajando para mejorarla. A priori las nuevas tecnologías y las redes sociales facilitan la difusión de la información y el acercamiento a las familias y tienen un papel cada vez más relevante, pero en la práctica, sobre todo ante información calificada de importante, se cree que es mejor combinar los canales tradicionales (circulares en papel, difusión oral, carteles, etc.) con los nuevos medios digitales (correo electrónico, blogs, WhatsApp, etc.).

Especialmente en las escuelas pequeñas, la comunicación informal (sobre todo en los momentos de entrada y salida) sigue destacando. En cambio, en los institutos, debido a que los adolescentes suelen ser mediadores-filtro entre el centro y las familias, la comunicación informal pasa a un segundo plano y se realza el papel de la comunicación digital, que se percibe como un buen medio, aunque mejorable, para informar y comunicarse con las familias.

Están intentando involucrar a las nuevas tecnologias con lo que es la comunicación con el resto de personas, se está trabajando en hacer una página web y unos medios de comunicación que sean más flexibles con las personas y la gente del instituto. Luego está la comunicación a través de las asambleas que son generales y abiertas a todos. (IPUA214AII).

Otros canales tradicionales son las reuniones y asambleas, como también señalan otros estudios (Garreta, 2008) pero con porcentajes relativamente bajos de asistencia ${ }^{7}$. Si bien en las escuelas la mención a los buzones de sugerencias o plafones informativos es más bien escasa, en los centros de secundaria ninguno de los entrevistados hace referencia a estos elementos. Un factor a tener en cuenta es que los padres no suelen llevar a sus hijos adolescentes al centro. Generalmente la participación de las familias en los centros de secundaria se produce de forma un tanto instrumental, los padres suelen acudir al centro cuando se les llama y sobre todo cuando se trata de algún aspecto importante relacionado con sus hijos.

Una particularidad de las AMPA es la temporalidad de sus miembros. Es decir, una persona solo puede ser socia del AMPA de un centro mientras su hijo estudia allí, lo que provoca una renovación de los socios cada curso y, a menudo, también de la junta. Esta característica tiene como aspecto positivo el flujo de ideas nuevas. Pero a la vez tiene aspectos negativos, como el hecho de que no se puedan continuar proyectos ya iniciados, la falta de comunicación entre la junta vieja y la nueva, la falta de información de los nuevos miembros, dificultades para encontrar personas voluntarias que se quieran involucrar y formar parte de las juntas, etc.

Cuando los padres entran nuevos, ¿no? Familias que entran nuevas, entran con mucho empije y muchas ganas, y lo quieren cambiar todo, y al cabo de dos cursos ya están quemados. (CPUPP014B).

Relacionado con este punto, otro reto señalado sobre todo por parte de los representantes asociativos es la participación en la junta, especialmente en el caso de las familias de origen inmigrante

7 Garreta (2008) señala que mientras que la media de inscripción al AMPA se sitúa en el 57,5\%, la de asistencia a las reuniones es del $18,3 \%$. 
y minorías étnicas (consultar Llevot y Bernad, 2016). Trasladan su visión de que entre unos pocos lo tienen que hacer todo y las dificultades para encontrar relevo para los cargos. Aunque en algunas AMPA el trabajo por comisiones, por ejemplo, está bien valorado, y se señalan una organización y un funcionamiento eficaces, en otras se consideran mejorables varios aspectos de funcionamiento y organización interna, como la distribución de las tareas y comunicación entre los miembros de la junta y el traspaso de información.

¿Cómo fue todo? Pues que en la reunión bubo tres o cuatro personas más de los normales, y nos pillaron. Directamente, eh? (Y rie). Es que yo me encontré de presidenta y no quería ser. (CPCA114A).

Hace tres, tres cursos que funciona, y entonces es un poco más de repartirse el trabajo, ¿no? Que cada uno se encarga de algo muy puntual, y asi queda el trabajo más repartido. Que no todo sobre la junta, ¿no? (CPUPP014B).

Resumiendo, algunos de los problemas a que se enfrentan las AMPA en el momento actual derivan de: la disminución del número de socios debido a problemas socioeconómicos y también al desconocimiento del papel de la asociación en el centro escolar; la baja implicación y participación en las actividades organizadas por las AMPA; y dificultades para renovar y mantener activamente las juntas de gobierno de la asociación. Así, los principales retos son: trabajar conjuntamente con el profesorado y con otros actores educativos para mejorar la participación de las familias y que esta revierta positivamente en la calidad del centro y de la educación de sus hijos e hijas; mejorar la organización y funcionamiento internos y también la comunicación con las familias, con los equipos directivos y profesorado y con el entorno; y establecer redes de trabajo colaborativo con otras AMPA del municipio o comarca.

\section{A modo de conclusiones}

Uno de los factores que favorecen la participación de las familias es la buena gestión y funcionamiento de las AMPA (Garreta, 2012; Llevot y Bernad, 2015). Los equipos directivos y representantes asociativos entrevistados piensan que un AMPA dinámica y participativa (en sintonía con el proyecto educativo de la escuela) favorece el funcionamiento del centro. Precisamente, en el contexto actual, algunas asociaciones manifiestan ser más activas y haber ampliado en los últimos años, con la complicidad de los equipos directivos, el repertorio de servicios y actividades que ofrecen a las familias y a los centros, quizás por la necesidad de paliar las deficiencias de la Administración y hacer más atractiva la escuela a los ojos de las familias, situadas, muchas de ellas, en el paradigma de los padres-clientes.

Teniendo en cuenta las funciones de las AMPA y las actuaciones que realizan en los centros, se podrían distinguir varios niveles de colaboración. En un primer nivel, se encontrarían las funciones de apoyo logístico y material al centro (compra de material deportivo, fungible, libros para la biblioteca, colaboración económica y en la organización de las fiestas de la escuela). En un segundo nivel, estaría la gestión de servicios (servicio de comedor, actividades extraescolares, etc.) y la organización de actividades educativas para el alumnado y sus familias (salidas de fin de semana, colonias, fiestas, escuela de padres, conferencias...). En un tercer nivel, la comunicación con el centro, especialmente las reuniones periódicas con la dirección, en las que se debate sobre las necesidades de la escuela y de los padres. En un cuarto nivel, la comunicación con las familias, la dinamización de la participación de las familias y la defensa de sus intereses (y los del centro). Por último, en un quinto nivel, habría la participación del AMPA en los órganos de gestión, es decir, en el Consejo Escolar y en otros, como las asambleas de delegados. 
Pero a veces algunas AMPA actúan en espacios intersticiales donde las fronteras simbólicas (y físicas) son difusas. Si bien las reivindicaciones ante la Administración relativas a obras, por ejemplo, son bien recibidas por los docentes, ya que, generalmente, son actuaciones de apoyo al centro y las asociaciones actúan como aliadas, cuando la iniciativa la emprenden las familias, y quizás en temas que se piensa que no son de su ámbito competencial (como la participación en el currículo o en el diseño de los proyectos de centro), los equipos directivos y las administraciones se pueden sentir amenazados y dejar de sentir a las asociaciones como aliadas, de modo que puede quebrarse el frágil equilibrio de las relaciones de poder, lo que puede significar el inicio de espirales negativas en la relación.

En cuanto al funcionamiento del AMPA, a pesar de que está constituida en la mayoría de centros y muchas familias son socias (aspecto valorado por los docentes y representantes asociativos), la participación real en muchos casos es decepcionante.

Las causas que dificultan la participación en las AMPA son difíciles de determinar, pero, sin ánimo de generalizar, nos atrevemos a señalar las siguientes:

Socioculturales: falta de tradición participativa y falta de cultura asociativa; experiencia escolar previa; dificultades lingüísticas.

Socioeconómicas: dificultad en compatibilizar el trabajo y las obligaciones familiares con la participación en la escuela; dificultad en pagar las cuotas.

Institucionales: desconocimiento de los órganos y canales de participación; canales de comunicación poco efectivos; percepción de una junta cerrada a la entrada de otros padres; conflictos; responsabilidad civil/penal que conlleva el cargo ejercido como miembro de la junta de AMPA; falta de liderazgo en la asociación y en el equipo directivo; actitudes del equipo directivo y del profesorado poco favorables a la participación de las familias; percepción de poca representatividad de la junta; temor a enfrentarse a la dirección del centro.

Personales: falta de compromiso personal a medio y largo plazo; percepción de pocos beneficios personales; infravaloración de las propias habilidades y aptitudes para desempeñar cargos.

Una de las fortalezas señaladas son las buenas relaciones establecidas con el profesorado, especialmente con el equipo directivo, y con las familias. Seguramente, la comunicación, la colaboración y confianza mutuas, el sentimiento de pertenencia, de apoderamiento del proyecto, que se siente como propio, y el clima familiar que suele reinar en estos centros son factores a tener en cuenta. También el hecho de participar en el AMPA sirve a las familias para conocer mejor el funcionamiento del centro y también para incrementar su capital social.

El AMPA tendría que jugar un papel importante en la dinamización de la participación de todas las familias de la escuela (Abelló, 2008), pero generalmente no es así, relegándose su papel a suministrar servicios a los padres (se detecta una relación clientelar) y al centro (enmendando las carencias de la Administración), aspectos, por otro lado, valorados por los docentes y también por los progenitores. Pensamos que hace falta que las AMPA redefinan su papel y busquen nuevas estrategias para abrirse y comunicarse con todas las familias del centro, sin excepción, y dar respuesta a sus intereses. Y en este punto, sobre todo en los centros de secundaria, las nuevas tecnologías son una herramienta a tener en cuenta, como demuestran algunas experiencias: páginas web, blogs, redes sociales, etc.

Es necesario que la junta del AMPA represente al conjunto de padres y madres y sea portavoz real de sus representados para que estos órganos sean auténticos foros de representación. Una de las condiciones es que la vida diaria del centro y de las aulas sea también democrática y participativa y se instauren otros 
canales de participación entre los diferentes sectores de la comunidad, más cercanos a los intereses de las familias y que facilitarían su implicación y una comunicación bidireccional. Difícilmente podemos hablar de participación democrática si no se democratiza a la vez la estructura de funcionamiento cotidiano del centro.

\section{Referencias bibliográficas}

Abelló, L. (2008): Las madres y los padres, en: R. Miralles (coord.) ¿Quién manda en la escuela? (Madrid, Wolters Kluwer).

Andrés, S. y Giró, J. (2016): El papel y la representación del profesorado en la participación de las familias en la escuela, Revista Electrónica Interuniversitaria de Formación del Profesorado, 19 (1) 61-71, <http:// dx.doi.org/10.6018/reifop.19.1.245461>.

Bolívar, A. (2006): Familia y escuela: dos mundos llamados a trabajar en común, Revista de educación, 339, 119-146.

Epstein, Joyce L. (2001): School, Family and Community Partnerships: Preparing Educators and Improving Schools (Boulder, Westview Press).

Frías del Val, A. S. (2014): Evolución del marco normativo español sobre la participación de las familias. Contexto histórico, en: Consejo Escolar del Estado (ed.) La participación de las familias en la educación escolar (Madrid, Ministerio de Educación, Cultura y Deporte).

Garreta, Jordi (2008): La participación de las familias en la escuela pública. Las asociaciones de madres y padres del alumnado (Madrid, CEAPA).

Garreta, J. (2009): Escuela y familias inmigradas: Relaciones complejas, Revista Complutense de Educación, 20 (2) $275-291$.

Garreta, Jordi (2012): Families i escoles. La participació de les families als centres educatius (Lleida, Ajuntament de Lleida).

Garreta, J. (2015): La comunicación familia-escuela en educación infantil y primaria, Revista de la Asociación de Sociología de la Educación (RASE), 8 (1)71-85.

Garreta, J. y Llevot, N. (2015): Family-school communication in Spain: channels and their use, Ehquidad international Welfare Policies and Social Work Journal, 3 29-48.

Garreta, J. (2016): Las asociaciones de madres y padres en los centros escolares de Cataluña: puntos fuertes y débiles, Revista electrónica interuniversitaria de formación del profesorado, 19 (1) 47-59, <http://dx.doi. org/10.6018/reifop.19.1.245641>.

Giró, J. y Andrés, S. (2014): Las familias se suben a la "Marea Verde". El papel de madres y padres en las movilizaciones educativas, Revista de la Asociación de Sociología de la Educación, 7 (3) 627-643.

Giró, J.; Mata, A.; Vallespir, J. y Vigo, B. (2014): Familias y escuelas: los diferentes discursos sobre la participación, Ehquidad International Welfare Policies and Social Work Journal, 2, 65-90.

Giró, J. y Andrés, S. (2015): Familias y escuelas: nuevas reivindicaciones en el contexto de crisis", en Actas Congreso Desigualdad y democracia: Políticas públicas e innovación social (Barcelona, Red Española de Política Social).

Grant, B. Kathy y Ray, A. Julie (2013): Home, School, and Community Collaboration (London, Sage). 
Llevot, N. y Bernad, O. (2015): La participación de las familias en la escuela: factores clave, Revista de la Asociación de Sociología de la Educación, 8 (1) 57-70.

Llevot, N. y Bernad, O. (2016): La mediación gitana: herramienta performativa de las relaciones entre escuela y familia, Revista electrónica interuniversitaria de formación del profesorado, 19 (1), 99-110, <http://dx.doi. org/10.6018/reifop.19.1.245581>.

Kherroubi, Martine (dir.) (2008): Des parents dans l'école (Ramonville Saint-Agne, Éditions Érès).

Macià, M. (2016): La comunicación familia-escuela: el uso de las TIC en los centros de Primaria, Revista electrónica interuniversitaria de formación del profesorado, 19 (1) 73-83, <http://dx.doi.org/10.6018/reifop.19.1.245841>.

Martínez González R. A.; Rodríguez, B. y Gimeno, J. L. (2010): Áreas de cooperación entre los centros docentes y las familias, Estudio de caso. Educatio Siglo XXI, 28 (1)127-156.

Serra, C. (2004): Etnografía escolar, etnografía de la educación, Revista de Educación, 334 165-176.

Vallespir, J.; Rincón, J. C. y Morey, M. (2016): La participación de las familias en el Consejo Escolar y la formación del profesorado, Revista Electrónica Interuniversitaria de Formación del Profesorado, 19 (1) 31-45, $<$ http://dx.doi.org/10.6018/reifop.19.1.245751>.

Vega, Ana María (coord.); Raya, Esther; Navaridas, Fermín; Giró, Joaquín; Muñoz, J. A. y Blandini, M. (2012): Indicadores de participación de los padres en la escuela: un enfoque innovador para una educación de calidad (Madrid, Wolters Kluwer España). 